\title{
DIFERENTES ÁREAS ADESIVAS AFETAM A RESISTÊNCIA DE UNIÃO AO MICROCISALHAMENTO?
}

\author{
Kevelin Poliana Palma Rigo Thiesen* \\ Mariana Macedo Ribas ${ }^{*}$ \\ Guilherme Schmitt de Andrade ${ }^{* * *}$ \\ Flávia Pardo Salata Nahsan ${ }^{* \star * *}$ \\ Vera Lucia Schmittt**** \\ Fabiana Scarparo Naufel ${ }^{*+\alpha+k+t}$
}

\begin{abstract}
RESUMO: Quanto menor a superfície da área, maior a resistência de união, pois, espécimes com pequena área de superfície apresentam melhor distribuição de estresse, bem como reduzido número de defeitos internos. 0 objetivo deste estudo foi verificar a resistência de união ao microcisalhamento de espécimes com dois diferentes diâmetros, $0.55 \mathrm{~mm}$ e $0.76 \mathrm{~mm}$. 40 fatias de cerâmica Suprinity, com 1,3 mm de espessura, polidas em lixa $\# 600$, foram divididas aleatoriamente em dois grupos $(n=20)$. Em cada fatia, quatro áreas adesivas foram delimitadas por fita adesiva, sendo então fixadas as matrizes circulares. 0s espécimes foram condicionados com ácido fluorídrico a 5\% (FGM) por $20 \mathrm{~s}$, lavados e secos; então silanizados (RelyX Ceramic Primer - 3M ESPE) e secos com jato de ar. As matrizes foram preenchidas com cimento resinoso RelyX U200 3M ESPE e fotopolimerizadas por $100 \mathrm{~s}$. Após $24 \mathrm{~h}$ em água deionizada a $37^{\circ} \mathrm{C}$, os espécimes foram submetidos ao teste de microcisalhamento sob velocidade de $0,5 \mathrm{~mm} / \mathrm{min}$. Os dados normais e homogêneos foram submetidos à ANOVA para o experimento inteiramente casualizado, não exibindo diferença significativa $(p=0,62)$ entre as duas áreas avaliadas $-23,74 \pm 4,49$ e 24,85 $\pm 4,41$, respectivamente para as áreas 0,55 e 0,76 mm. Concluímos que, não há diferença na resistência de união ao microcisalhamento quando se utilizam as diferentes áreas adesivas estudadas.
\end{abstract}

PALAVRAS-CHAVE: Resistência ao cisalhamento; Adesivos; Cerâmicas.

\section{DIFFERENT ADHESIVE AREAS AFFECT RESISTANCE TO MICRO-SHEARING BONDING?}

ABSTRACT: The smaller the surface area, the greater is the resistance. Specimens with small surface area have a better stress distribution and a reduction in the number of internal faults. Bonding resistance to micro-shearing of specimens with two different diameters, $0.55 \mathrm{~mm}$ and $0.76 \mathrm{~mm}$, were analyzed. Forty Suprinity ceramic slices, $1.3 \mathrm{~mm}$ thick, polished by file \#600, were randomly divided into two groups $(n=20)$. Four adhesive areas in each slice were delimited by adhesive tape and fixed to circular matrixes. Specimens were conditioned with hydrofluoric acid 5\% (FGM) for $20 \mathrm{~s}$, washed, dried, silanized (RelyX Ceramic Primer - 3M ESPE) and dried by air jet. Matrixes were filled with resinous cement RelyX U200 3M ESPE and photopolymerized for $100 \mathrm{~s}$. After $24 \mathrm{~h}$ in de-ionized water at $37^{\circ} \mathrm{C}$, the specimens underwent micro-shearing test at $0.5 \mathrm{~mm} / \mathrm{min}$. Normal and homogeneous data were submitted to ANOVA for totally randomized assay, with no significant difference $(p=0.62)$ between the two areas under analysis $-23.74 \pm 4.49$ and $24.85 \pm 4.41$, respectively for areas 0.55 and $0.76 \mathrm{~mm}$. Results show that no bonding resistance to micro-shearing exists when different adhesive areas are employed.

KEYWORDS: Resistance to shearing; Adhesives; Ceramics.

\footnotetext{
Universidade Estadual do Oeste do Paraná (UNIOESTE) Departamento de Odontologia Restauradora, Divisão de Materiais Dentários, Brasil. E-mail: poly_rpk@hotmail.com

** Universidade Estadual do Oeste do Paraná (UNIOESTE). Departamento de Odontologia Restauradora, Divisão de Materiais Dentários, Brasil.

*** Universidade Estadual Paulista (UNESP) Instituto de ciência e tecnologia, Departamento de prótese e materiais dentários, São José dos Campos (SP), Brasil.

${ }^{* * * *}$ Universidade Federal de Sergipe (UFS). Docente Adjunta, Programa em Odontologia, Brasil.

${ }^{* * * * *}$ Universidade Estadual do Oeste do Paraná (UNIOESTE). Departamento de Odontologia Restauradora, Divisão de Materiais Dentários, Brasil.

${ }^{* * * * * * *}$ Universidade Estadual do Oeste do Paraná (UNIOESTE). Departamento de Odontologia Restauradora, Divisão de Materiais Dentários, Brasil.
} 


\section{INTRODUÇÃo}

A efetiva união adesiva entre dente e material restaurador tem sido otimizada de forma constante, em função do desenvolvimento de técnicas e materiais restauradores adesivos. A performance e a longevidade desta união pode ser verificada por meio de testes de resistência de união, que podem ser macro ou micro, dependendo do tamanho da área de união (SIRISHA et al., 2014a; VAN MEERBEEK et al., 2010). Os testes de resistência de união macro, ou seja, com uma área maior que $3 \mathrm{~mm}^{2}$ podem ser cisalhamento, tração ou push-out (VAN MEERBEEK et al., 2010; SIRISHA et al., 2014b).

Os macro testes de tração foram utilizados por muito tempo para mensurar e caracterizar 0 comportamento das interfaces adesivas. Com 0 passar dos anos, pesquisadores perceberam que amplas áreas de superfície produziam falhas coesivas (PASHLEY et al., 1995; PHRUKKANON; BURROW; TYAS, 1998) e baixa resistência de união, onde em áreas menores que $2 \mathrm{~mm}^{2}$, as falhas eram adesivas e apresentavam alta resistência de união (PASHLEY et al., 1995; PHRUKKANON; BURROW; TYAS, 1998; SANO et al., 1994). Observou-se que a resistência de união aumentava exponencialmente, quando diminuía a área de superfície. A razão para este fenômeno poderia ser pela presença de defeitos e/ou tensão concentrada na interface e/ou no substrato, explicado pela Griffith's defect theory em que a resistência à tração do material diminuiu com o aumento do tamanho do espécime (SANO et al., 1994).

Os testes realizados para configurar a relação entre área de superfície e resistência de união foram feitos com secção transversal quadrada, porém, pela desigualdade na distribuição de estresse na interface adesiva, foi proposto avaliar a resistência de união ao cisalhamento e à tração com espécimes de secção transversal circular (PHRUKKANON; BURROW; TYAS, 1998). Pela dificuldade na confecção dos espécimes, a menor área de união utilizada foi de $1.1 \mathrm{~mm}^{2}$. Para tanto, Shimada,Yamaguchi e Tagami (2002) apresentaram uma metodologia eficiente para a execução de testes de cisalhamento em nível micro, caracterizado como microcisalhamento ( $\mu \mathrm{SBS})$.

Com o intuito de avaliar a resistência da união adesiva entre compósito resinoso e cerâmica, a partir da tensão de cisalhamento, os autores utilizaram de tubos tygon, com diâmetro aproximado de 0,75 mm, preenchidos com resina composta e posicionados em três ou quatro locais diferentes na superfície cerâmica. De acordo com os autores poderia ter sido utilizado o teste de microtração, no entanto, consumiria muito tempo e seria difícil confeccionar as amostras, principalmente por serem cerâmicas vítreas (SHIMADA; YAMAGUCHI; TAGAMI 2002). Após este trabalho, a comunidade científica passou a aplicar esta metodologia na avaliação da resistência de união ao microcisalhamento.

Todavia, as dificuldades na obtenção do tubo tygon, na fixação durante 0 desenvolvimento da metodologia e na remoção após polimerização do material, Tedesco et al. (2013) levaram à busca por outros materiais, com a capacidade de servir como matriz para a confecção de corpos de prova com diâmetro e altura compatível ao que requeria a técnica de microcisalhamento. Desta forma, foram utilizados matriz de silicone (VIEIRA, 2013; MORAES et al., 2008), tubos de amido (TEDESCO et al., 2013), tubos de plástico (PHARK et al., 2009) e placas de metal perfuradas e justapostas (GIANCHETTI, 2012).

No presente trabalho foi utilizado um cateter intravenoso à base de teflon (Solidor, Haryana, Índia), para comparar dois diâmetros diferentes: $0,55 \mathrm{~mm}$ e 0,76 mm e verificar se há diferença expressiva na resistência de união à cerâmica pelo teste de microcisalhamento.A hipótese nula éa de que diâmetros diferentes de corpo-de-prova não interferem na resistência de união ao microcisalhamento. 


\section{METODOLOGIA}

Este estudo foi conduzido in vitro e envolveu 0 fator com diferentes diâmetros da matriz para confecção do espécime. A variável de resposta usada foi analisada por meio do teste de microcisalhamento.

Foi confeccionado dois cilindros de alumínio com diâmetro de $1 \mathrm{~cm}$ e espessura de $8 \mathrm{~mm}$ com um orifício ao centro nos diâmetros de $0.9 \mathrm{~mm}$ e $1.1 \mathrm{~mm}$ para otimizar 0 corte do cateter. 0s tubos entraram justapostos à parede do orifício e com uma lâmina de bisturi \#15 foi possível cortá-lo de forma perpendicular em um ângulo de aproximadamente $90^{\circ}$ (Figura 1).

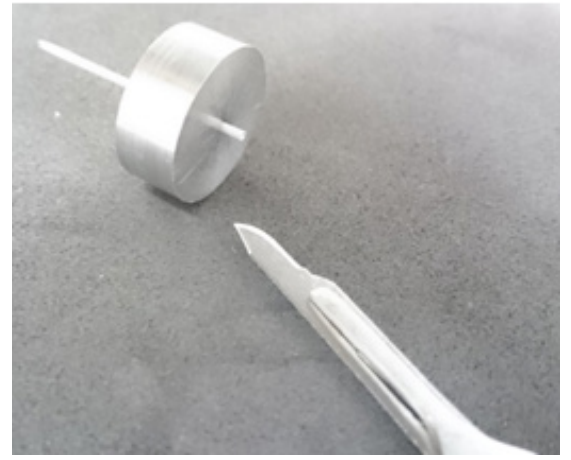

Figura 1. Imagem ilustrativa do dispositivo utilizado para corte do cateter

Blocos da cerâmica Suprinity foram cortados (Labcut 1010 extec, Enfield, USA) em fatias com as dimensões de $12 \times 7 \mathrm{~mm}$ e $1,4 \mathrm{~mm}$ de espessura, sendo cristalizados de acordo com 0 fabricante no forno Vita Vacumat 6000M (Vita Zahnfabrik, Bad Säckingen, Germany). Estes foram fixados em anel de PVC preenchido com resina acrílica por meio de resina epóxi, lixados na politriz (Arotec PL4, São Paulo, Brazil) durante 1 min com lixa d'água grit \# 100, 320 e 600, respectivamente, e distribuídos aleatoriamente em dois grupos.

Todas as superfícies cerâmicas foram então condicionadas com ácido fluorídrico $5 \%$ (Condac porcelana, FGM, Joinville, Brazil) durante 20 s e lavadas em água abundante por $1 \mathrm{~min}$. Com um perfurador de Ainsworth, pedaços de fita isolante (Imperial, 3M, Sumaré, Brazil) nas cores azul e vermelha foram perfurados com quatro furos equivalentes para cada diâmetro. A fita azul, para o tubo com diâmetro interno de $0.55 \mathrm{~mm}$ e a fita vermelha, para 0 diâmetro interno de $0.76 \mathrm{~mm}$ (Figura 2).

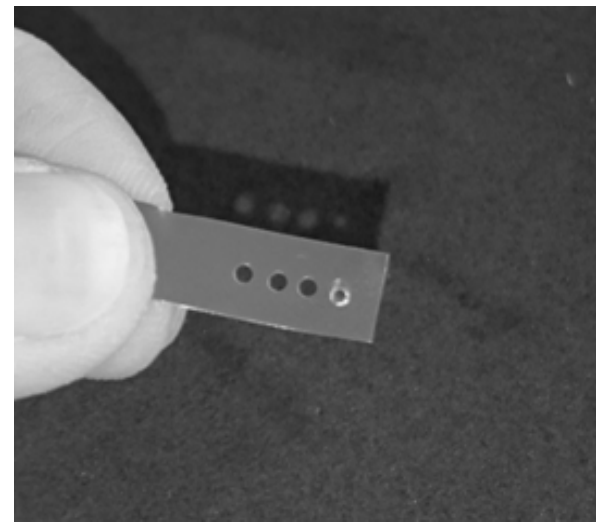

Figura 2. Imagem ilustrativa da fita perfurada. Observa-se a presença da matriz de teflon adaptada à última perfuração com redução do diâmetro da luz, vista a partir da sombra

Após delimitação da área adesiva pela fixação das fitas sobre a superfície cerâmica, foi aplicado Silano (RelyX Ceramic Primer, 3M ESPE, St Paul, USA) e ativado por $5 \mathrm{~s}$, secado com jato de ar e aplicada a segunda camada. Após a silanização, os tubos de teflon foram preenchidos com cimento resinoso RelyX U200 (3M ESPE, St Paul, USA), encaixados nas perfurações e fotopolimerizados por $100 \mathrm{~s}$ (Figura 3).

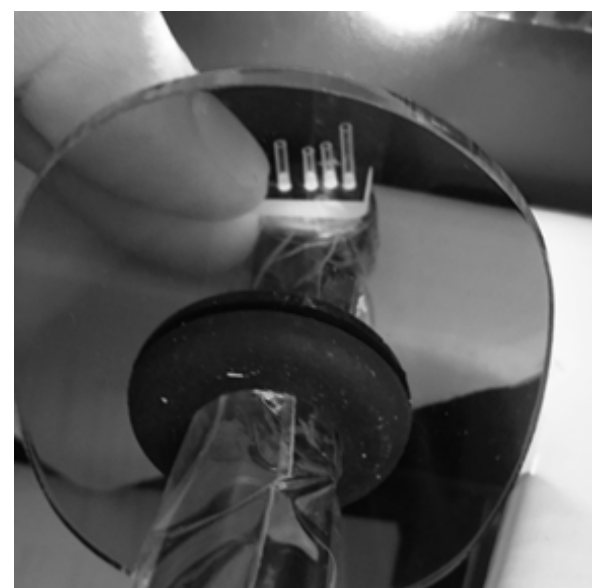

Figura 3. Imagem ilustrativa das matrizes de teflon preenchidas com cimento resinoso e fotopolimerizadas

Os espécimes foram armazenados em água deionizada a $37^{\circ} \mathrm{C}$, por $24 \mathrm{~h}$, previamente à remoção dos tubos de teflon e a remoção da fita isolante. Os 
corpos de prova foram então submetidos ao teste de microcisalhamento em uma máquina universal de ensaios (EMIC DL2000, São José dos Pinhais, Brazil) por meio de uma alça feita com fio ortodôntico $(0.20$ $\mathrm{mm}$ de diâmetro) ao redor do cilindro de resina. A força de cisalhamento foi então aplicada à velocidade de $0.5 \mathrm{~mm} / \mathrm{min}$ até ocorrer fratura.

Os valores em $\mathrm{N}$ foram divididos pela área da secção transversal, obtendo valores em MPa de resistência de união. A área da secção transversal foi determinada dividindo 0 diâmetro do tubo em milímetros pelo $\varpi(3,14)$ vezes o raio ao quadrado.

Os dados foram analisados estatisticamente pelos testes de Shapiro-Wilk (W), Anova e teste complementar de Tukey.

\section{RESULTADO}

Neste experimento inteiramente casualizado, os testes estatísticos mostraram que não houve diferença significativa entre os dois diâmetros de tubos testados, em que " $p$ " foi maior que $5(p=0.6233)$.

A resistência de união média em MPa para 0 grupo com diâmetro de $0,55 \mathrm{~mm}$ foi de $23,74 \pm 4,49$ e para 0 grupo com diâmetro de $0,76 \mathrm{~mm}$ foi de

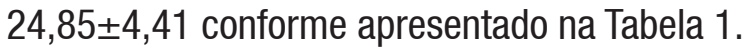

Tabela 1. Média (DP) da resistência de união do cimento resinoso à cerâmica Suprinity em dois diâmetros diferentes

\begin{tabular}{lll}
\hline Diâmetro & HSBS \\
\hline $0,55 \mathrm{~mm}$ & $23,74 \pm 4,49 \mathrm{a}$ & \\
$0,76 \mathrm{~mm}$ & $24,85 \pm 4,41 \mathrm{a}$ & \\
\hline Letras diferentes & sinalizam diferenças & estatisticamente \\
significantes & &
\end{tabular}

Na análise do modo de fratura realizada com microscópio digital (Dino-Lite Premier, Anmo Eletronics Corporation, New Taipei City, Taiwan) em um aumento de aproximadamente $240 \mathrm{x}$, foi observado que para os espécimes com diâmetro de 0,55 mm 0 tipo de fratura foi $100 \%$ adesivo (Figura 4), no entanto, para os espécimes com diâmetro de 0,76 mm o tipo de fratura foi $75 \%$ adesivo (Figura 5) e 25\% misto (Figura 6).

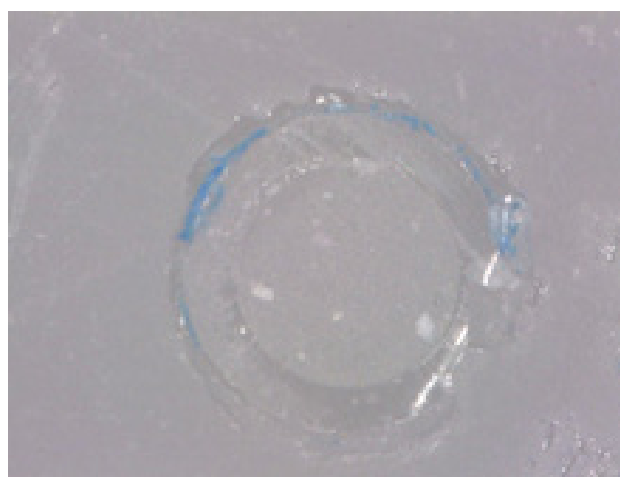

Figura 4. Imagem ilustrativa para fratura do tipo adesiva no diâmetro 0,55 mm

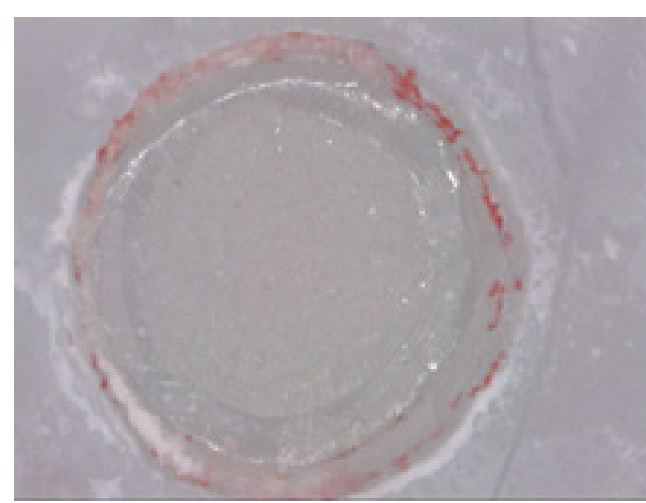

Figura 5. Imagem ilustrativa para fratura do tipo adesiva no diâmetro de 0,76 mm

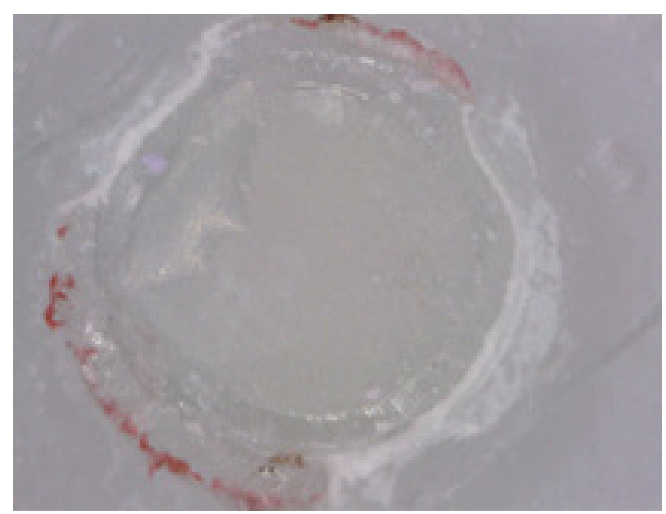

Figura 6. Imagem ilustrativa para fratura do tipo mista no diâmetro de 0,76 mm 


\section{DISCUSSÃo}

Este estudo avaliou a $\mu$ SBS de diferentes diâmetros de corpos-de-prova unidos à cerâmica Suprinity. Os resultados deste estudo aceitam a hipótese nula, em que não houve diferença estatística na resistência de união entre diferentes diâmetros.

0 teste de microcisalhamento foi desenvolvido (MCDONOUGH; ANTONUCCl; DUNKERS, 2001) e aprimorado (SHIMADA; YAMAGUCHI; TAGAMI, 2002), verificando que quanto menor a área mais preciso é o resultado (PASHLEY et al, 1995; PHRUKKANON; BURROW; TYAS, 1998). Portanto, a comunidade científica se desafiou e induziu novos pesquisadores a criar corpos-de-prova cada vez menores (SANO et al., 1994). Em estudos prévios observou-se que quando a área de superfície diminuía ocorria aumento na resistência de união e 0 modo de fratura era adesivo (PASHLEY et al, 1995; PHRUKKANON; BURROW; TYAS, 1998; SANO et al., 1994), porém, 0 menor diâmetro utilizado para o corpo de prova foi de 1.2 mm (PHRUKKANON; BURROW; TYAS, 1998). Nosso estudo mostrou que não houve aumento na resistência de união quando comparados dois diâmetros menores que $0.8 \mathrm{~mm}$, porém, para a análise no tipo de fratura observamos que 0 diâmetro de 0,76 mm apresentou maior quantidade de fratura mista $(25 \%)$ enquanto 0 tubo com diâmetro de 0,55 mm apresentou tipo de fratura $100 \%$ adesiva.

A menor área de superfície ou 0 menor diâmetro encontrado em estudos prévios foi de 0,75 mm (SHIMADA; YAMAGUCHI; TAGAMI, 2002). Neste estudo, o menor diâmetro utilizado foi de $0,55 \mathrm{~mm}$, em que observamos maior dificuldade na precisão do corte do tubo, na execução da metodologia, perdendo maior quantidade de corpos-de-prova, quando comparado ao diâmetro de $0,76 \mathrm{~mm}$, por defeitos de posicionamento e de cimentação. Talvez as dificuldades na execução destes testes de microcisalhamento levem a comunidade científica a retornar para diâmetros maiores (GIRALDO et al., 2016; CEKIC-NAGAS et al., 2016).

Para que os testes que estavam sendo realizados pudessem ser comparados entre si, foi necessário padronizar internacionalmente os métodos dos testes de adesão à estrutura dental, desta forma a ISO (International Standardization Organization) documentou as especificações técnicas pela primeira vez em 1994 como um relatório técnico (TR). Este relatório técnico evoluiu em 2003 para uma especificação técnica (TS) que foi revisada em 2015, sendo a padronização mais atual para metodologia dos testes de união adesiva entre materiais dentários e estrutura dental (ISO/TS 11405:2015 Dentistry Testing of adhesion to tooth structure).

De acordo com as exigências da ISO é necessário restringir a área adesiva para os testes de microcisalhamento. Em um trabalho em que se avaliou a influência da delimitação da área adesiva para este tipo de teste, observou-se que a delimitação da área adesiva resultou em valores baixos de resistência de união quando comparados ao grupo sem delimitação da área (SHIMAOKA et al., 2011). No entanto, esta adesão foi realizada em estrutura dentinária, em que se observou a penetração do sistema adesivo nos túbulos dentinários.

Neste estudo a delimitação da área foi feita, porém, os tubos de teflon encaixaram dentro da área delimitada, onde 0 operador conseguia sentir na mão quando o tubo estava sobre a fita ou sobre a superfície cerâmica, diferentemente da técnica executada com tubos tygon (SHIMADA; YAMAGUCHI; TAGAMI, 2002) ou de amido (TEDESCO et al., 2013), em que a luz do tubo necessita coincidir com o furo da fita fixada sobre a cerâmica, quase "as cegas". Além de que, para esta metodologia foi possível colocar o cimento no tubo e fixar sobre a cerâmica para diminuir a chance de desenvolver bolhas, diferentemente das técnicas empregadas para os tubos tygon (SHIMADA; YAMAGUCHI; TAGAMI, 2002) e amido (TEDESCO et al., 2013), em que estes são previamente fixados e, posteriormente, injetado o compósito resinoso, aumentando a chance de formação de bolhas e defeitos de cimentação.

Outro fator que pode interferir na utilização ou não da delimitação da área adesiva é a espessura da 
parede da matriz tubular utilizada para a confecção do corpo-de-prova. Podemos pressupor que se a espessura da parede do tubo ser de $2 \mathrm{~mm}$, a deposição de adesivo será muito maior do que se a parede tiver espessura de 0,1 mm. Em nosso trabalho, teorizamos que uma espessura de parede de 0,1 mm não foi capaz de interferir nos resultados, no entanto, mais estudos sobre a delimitação da área devem ser realizados em diferentes substratos para verificar qual 0 efeito do extravasamento do adesivo na resistência de união.

De forma consensual, alguns autores (PASHLEY et al., 1995; PHRUKKANON; BURROW; TYAS, 1998; SANO et al., 1994) justificaram que o tamanho da área de superfície interfere diretamente na resistência de união a ser testada. Eles argumentaram que quanto maior a área, maior o conteúdo de defeitos, que ao ser aplicada a força, a fratura pode iniciar nestes defeitos levando à distribuição não uniforme de estresse e ruptura da interface de união.

Uma das principais vantagens desta metodologia foi a remoção do tubo, pois, após polimerização e armazenamento em água por 24 h, a matrizéfacilmente removida por delicado tracionamento manual, sem exercer tensão sobre 0 corpo-de-prova cimentado, nem tampouco necessitando ser cortado. Em um estudo que compara o uso de tubos tygon e de tubos de amido na confecção de corpos-de-prova para microcisalhamento, em que após armazenamento em água os tubos por si só se soltavam, observou-se que não houve diferença significativa na resistência de união para o fator "tipo de tubo", no entanto, observamos que a resistência de união em MPa diminuiu em cerca de $15 \%$ quando o tubo de amido foi utilizado (TEDESCO et al., 2013). Talvez, a absorção de água e solventes pelas paredes de amido do tubo tenha interferido no escoamento do compósito criando bolhas e diminuindo o embricamento mecânico, no entanto, estas são especulações que devem ser verificadas.

Esta metodologia foi executada de forma simples e eficiente para verificar a resistência de união entre cerâmica e cimento resinoso em área de superfície ineditamente pequena. Como todas as metodologias pode ter seus viéses, que podem ser aceitos desde que sejam padronizados para todo 0 grupo amostral.

\section{CONCLUSÃO}

Concluímos que 0 uso de diferentes diâmetros dos corpos-de-prova não influenciou na resistência de união entre cimento resinoso e cerâmica Suprinity.

\section{REFERÊNCIAS}

CEKIC-NAGAS, I. et al. Micro-shear bond strength of different resin cements to ceramic/glass-polymer CAD-CAM block materials. J Prosthodont Res, 2016.

GIACHETTI, L. et al. Reparability of aged silorane with methacrylate-based resin composite: micro-shear bond strength and scanning electron microscopy evaluation. Oper Dent, p. 28-36, 2012.

GIRALD0, T.C. et al. Active and Passive Application of the Phosphoric Acid on the Bond Strength of Lithium Disilicate. Braz Dent J, p. 90-94, 2016.

MCDONOUGH, W.G.; ANTONUCCI, J.M.; DUNKERS, J.P. Interfacial shear strengths of dental resin-glass fibers by the microbond test. Dent Mater, p. 492-498, 2001.

MORAES, R.R. et al. Light-activation of resin cement through ceramic: relationship between irradiance intensity and bond strength to dentin. J Biomed Mater Res B ApplBiomater, p. 160-165, 2008.

PASHLEY, D.H. et al. Adhesion testing of dentin bonding agents: a review. Dent Mater, p. 117-125, 1995.

PHARK, J.H. et al. An in vitro evaluation of the longterm resin bond to a new densely sintered high-purity zirconium-oxide ceramic surface. J Prosthet Dent, $p$. 29-38, 2009.

PHRUKKANON, S.; BURROW, M.F., TYAS, M.J. Effect of cross-sectional surface area on bond strengths between resin and dentin. Dent Mater, p. 120-128, 
1998.

SANO, H. et al. Relationship between surface area for adhesion and tensile bond strength--evaluation of a micro-tensile bond test. Dent Mater, p. 236-240, 1994.

SHIMADA, Y.; YAMAGUCHI, S.; TAGAMI, J. Micro-shear bond strength of dual-cured resin cement to glass ceramics. Dent Mater, p. 380-388, 2002.

SHIMAOKA, A. M. et al. The importance of adhesive area delimitation in a microshear bond strength experimental design. J Adhes Dent, p. 307-314, 2011.

SIRISHA, K. et al. Validity of bond strengthtests: A critical review: Part I. J Conserv Dent, p. 305-311, 2014a.

SIRISHA, K. et al. Validity of bond strengthtests: A critical review-Part II. J Conserv Dent, p. 420-426, 2014b.

TEDESCO, T. K. et al. Starchtubing: an alternative method to build up microshear bond test specimens. $\mathbf{J}$ Adhes. Dent, p. 311-315, 2013.

VAN MEERBEEK, B. et al. Relationship between bondstrength tests and clinical outcomes. Dent Mater, $p$. 100-121, 2010.

VIEIRA, H. H. Influência do tipo de matriz e de um antioxidante experimental na resistência de união entre compósito e estruturas dentais através de microcisalhamento. 2013. Dissertação (Mestrado em Odontologia) - Faculdade de Odontologia de Piracicaba, Piracicaba, SP.

Recebido em: 07 de agosto de 2017 Aceito em: 30 de outubro de 2017 